\title{
Estructura poblacional y proporción de sexos en Caiman crocodilus en Caño Negro, Costa Rica
}

\author{
Armando H. Escobedo-Galván
}

Escuela de Ciencias Biológicas, Universidad Nacional, Heredia, Costa Rica. Dirección actual: Laboratorio de Análisis Espaciales, Instituto de Biología, Universidad Nacional Autónoma de México, 3er Circuito Exterior s/n, Ciudad Universitaria, C.P. 04510, México D.F. (elchorvis@gmail.com)

\begin{abstract}
Population structure and sex ratio in Caiman crocodilus in Caño Negro, Costa Rica. Evaluation of population status of Caiman crocodilus (Linnaeus, 1758) in Caño Negro Wildlife Refuge started in 1986. However, only information related with abundance and size of the population is known. The objective of this study was to evaluate population structure and sex ratio of $C$. crocodilus. We captured 145 caimans in 22 nocturnal spotlight surveys from May/2004 to May/2005. Most of caimans were less than $60 \mathrm{~cm}$ in length and between 150 to $180 \mathrm{~cm}$. The average sex ratio was 1:6.06 female/male, being one of the highest differences reported for this species. If this sex ratio persists, there is going to be a decrease in the population viability in a short and long term period.
\end{abstract}

KEYWORDS. Caiman crocodilus, population structure, sex ratio, Caño Negro, Costa Rica.

RESUMEN. Desde 1986 se han llevado acabo evaluaciones sobre el estado poblacional de Caiman crocodilus (Linnaeus, 1758) en el Refugio Nacional de Vida Silvestre Caño Negro. Sin embargo, solo se conoce información sobre la abundancia y el tamaño poblacional. El objetivo de este estudio fue evaluar la estructura poblacional y proporción de sexos de C. crocodilus en Caño Negro. Se capturó un total de 145 caimanes mediante 22 conteos nocturnos desde Mayo 2004 a Mayo 2005. La mayoría de los individuos presentaron longitudes menores a $60 \mathrm{~cm}$ y entre 150 a $180 \mathrm{~cm}$. La proporción de sexos promedio fue de 1: 6.06 hembra/machos, siendo una de las mayores diferencias reportadas para la especie; de mantenerse la proporción de sexos, es de esperar una disminución en la viabilidad poblacional a corto y mediano plazo.

PALABRAS ClAVES. Caiman crocodilus, estructura poblacional, proporción de sexos, Caño Negro, Costa Rica.

El caimán, Caiman crocodilus (Linnaeus, 1758), es la especie más abundante y de mayor ámbito de distribución de los crocodilianos neotropicales (Ross, 1998). Sin embargo, la explotación ilegal y la pérdida de hábitat en toda su área de distribución, han provocado que las poblaciones de caimanes disminuyan el número de individuos óptimo para mantener algunas de sus poblaciones viables a largo plazo. Esto ha generado la necesidad de obtener información sobre el estado de las poblaciones con la finalidad de establecer políticas de manejo y áreas prioritarias de conservación en algunas especies de cocodrilos (THORBJARNARSON et al., 2006).

Campos et al. (1994) mencionaron que para el establecimiento de planes de manejo, in situ y ex situ, dirigidos a la conservación de las poblaciones de caimanes se requiere de información referente a la distribución geográfica, la estructura poblacional, la dinámica poblacional y el desempeño biológico. El análisis de la estructura poblacional y la proporción de sexos permite conocer la tendencia de las poblaciones debido al impacto sufrido por la explotación comercial legal e ilegal en caimanes (Velasco \& Ayarzaguena, 1995; Mourao et al., 1996).

El objetivo de este estudio fue evaluar la estructura poblacional y proporción de sexos del caimán en el Refugio Nacional de Vida Silvestre Caño Negro, localizado en la zona norte de Costa Rica y hacer una comparación con los datos obtenidos por otros autores para la misma zona en años anteriores.

\section{MATERIAL Y MÉTODOS}

El estudio se lleva acabo en el Refugio Nacional de Vida Silvestre Caño Negro, sobre el cauce principal del río Frío, en la zona norte de Costa Rica $\left(10^{\circ} 54^{\prime} \mathrm{N}\right.$ y $\left.84^{\circ} 47^{\prime} \mathrm{W}\right)$. El refugio cuenta con una extensión de 9,969 ha y se caracteriza por ser un sistema de lagunas estacionales que varían dependiendo los cambios en el cauce principal del río Frío y sus afluentes, siendo una zona de regulación de inundaciones en la zona norte del país, ayudando a mantener la calidad ambiental (CASTILLO \& MARCH, 1993). CABRERA et al. (2003) mencionaron que la época seca va desde enero a abril y la época lluviosa de mayo a diciembre. Durante la época seca los niveles del agua descienden hasta transformar las lagunas en pequeños cuerpos de agua con poca profundidad perdiendo comunicación entre sí. Las lagunas presentan pendientes leves con valores inferiores al $5 \%$ y se sitúan por debajo de los 40 m.s.n.m. (CASTILLO \& MARCH, 1993).

Se realizaron 22 muestreos nocturnos en un bote de aluminio con motor fuera de borda (25 HP), entre los sectores San Sebastián y Playuelas del refugio, desde Mayo del 2004 a Mayo del 2005. Los recorridos tuvieron una duración de cinco horas, comenzando a las 19:00 h. Se utilizó la metodología del conteo visual nocturno, ubicando a los animales por el brillo de sus ojos utilizando lámparas encandiladoras de 4.8 voltios. Una vez localizado el animal, la captura consistió en tomar con la mano a individuos menores de $1.30 \mathrm{~m}$ y para tallas más grandes o animales ubicados en áreas de difícil acceso se utilizó un tubo de PVC de dos metros de largo con una soga insertada (SÁNCHEZ et al., 1996). Para evitar contar un individuo dos veces se utilizó el método propuesto por Bolton (1989) para la identificación de cocodrilos, el cual consiste en la corta de escamas caudales, otorgándoles una numeración con base en centenas, decenas y unidades; además se colocaron marcas de 
color (Cable tie) en la primera escama caudal simple para la diferenciación de sexos: rojo para hembras y blanco para machos. Para determinar el sexo, se utilizó el método directo de palpación por medio de la cloaca (BRAZAITIS, 1968) solo en individuos mayores a $40 \mathrm{~cm}$ de longitud total.

A cada uno de los individuos se le midió la longitud total ventral que va desde la punta del hocico hasta la punta de la cola. Para el análisis, se utilizaron dos clasificaciones con base a las tallas de los individuos capturados. En la primera fueron agrupados en clases cada $30 \mathrm{~cm}$ a partir de $10 \mathrm{c} 60 \mathrm{~cm}$ de longitud total para comparar la proporción de sexos observada en el presente estudio y la observada por E. F. Junier (datos no publicados), entre 1993 y 1994, en Caño Negro (Clase I $<60 \mathrm{~cm}$; Clase II = 60.1 a 90; Clase III = 90.1 a 120; Clase IV $=120.1$ a 150; Clase V = 150.1 a 180; Clase VI=180.1 a 210; Clase VII $>210.1 \mathrm{~cm})$. En la segunda se utilizó la clasificación de tamaños empleada por Velasco \& Ayarzaguena (1995) para conocer el efecto de la caza sobre la especie (Clase I $<50 \mathrm{~cm}$; Clase II $=50.1$ a $120 \mathrm{~cm}$; Clase III = 120.1 a $180 \mathrm{~cm}$; Clase IV = $180.1 \mathrm{~cm}$ ).

Se utilizó la prueba Chi-cuadrado para evaluar diferencias en el número de individuos por clase. Diferencias entre la proporción de sexos por clase fue evaluada mediante la prueba no paramétrica de MannWhitney. Variaciones estacionales en la proporción de sexos fueron evaluadas mediante una prueba ANOVA de dos vías. Por último, se utilizó la prueba de Chi-cuadrado para determinar si existieron diferencias entre la proporción de sexos por clase observados en este estudio y el realizado anteriormente, entre los años de 1993 y 1994, por E. F. Junier en Caño Negro (com. pers.).

\section{RESULTADOS}

Se capturó un total de 145 individuos en 22 muestreos; sin embargo, solo a 113 individuos capturados se les determinó el sexo debido al tamaño. Las clases I y $\mathrm{V}$ presentaron el mayor número de individuos capturados $\left(\chi^{2}=29.84 ; P<0.01 ;\right.$ Fig. 1$)$. El $85.84 \%$ de las capturas fueron machos, observándose diferencias en el numero de machos y hembras por clase $(U=57 ; P<0.01 ;$ Fig. 2$)$. La proporción de sexos por clases varió entre 1:2 y 1:22 hembra/macho, siendo la proporción de sexos promedio de 1:6.06 hembra/macho. No se observó una diferencia en el número de machos y hembras capturados entre época seca y lluviosa, siendo la interacción del número medio de individuos entre épocas y sexo, no significativo, mostrando una mayor media de machos capturados durante ambas épocas $(F=0.01 ; P>0.05)$. Además, se observó una diferencia en la proporción de sexos encontrada en el presente estudio y la observada entre 1993 y 1994 en Caño Negro para cada clase (Tab. I).

Con base a la clasificación de tallas utilizada por Velasco \& Ayarzaguena (1995), se observaron diferencias en la distribución del número de individuos por clase, siendo mayor el número de individuos en la clase III $\left(\chi^{2}=16.27 ; P<0.05\right)$. Se observaron diferencias en el número de individuos por clase de los 22 recorridos en comparación con el número de individuos capturados entre 1993 y 1994 para la misma zona $\left(\chi^{2}=51.52 ; P<0.01\right.$; Fig. 3).
Tabla I. Comparación de la proporción de hembra / macho de Caiman crocodilus (Linnaeus, 1758) por clase entre este estudio y los valores calculados anteriormente (1993 y 1994) en el Refugio Nacional de Vida Silvestre Caño Negro, Costa Rica.

\begin{tabular}{lccc}
\hline Clases & $\begin{array}{c}\text { Intervalo } \\
(\mathrm{cm})\end{array}$ & Este estudio & $\begin{array}{c}\text { E. F. Junier (datos } \\
\text { no publicados) }\end{array}$ \\
\hline Clase I & $<60$ & $1: 3.00$ & $1: 1.37$ \\
Clase II & $60-90$ & $1: 7.00$ & $1: 1.69$ \\
Clase III & $90-120$ & $1: 2.00$ & $1: 0.46$ \\
Clase IV & $120-150$ & $1: 22.00$ & $1: 0.75$ \\
Clase V & $150-180$ & $1: 5.80$ & - \\
Clase VI & $180-210$ & $1: 11.50$ & - \\
Promedio & & $1: 6.06$ & $1: 1.24$ \\
\hline
\end{tabular}

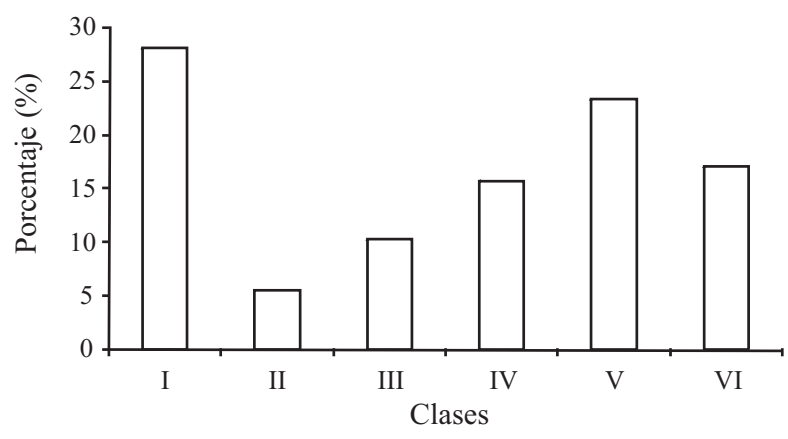

Fig. 1. Estructura poblacional de caimanes, Caiman crocodilus (Linnaeus, 1758), en Caño Negro, Costa Rica ( $\mathrm{n}=145)$.

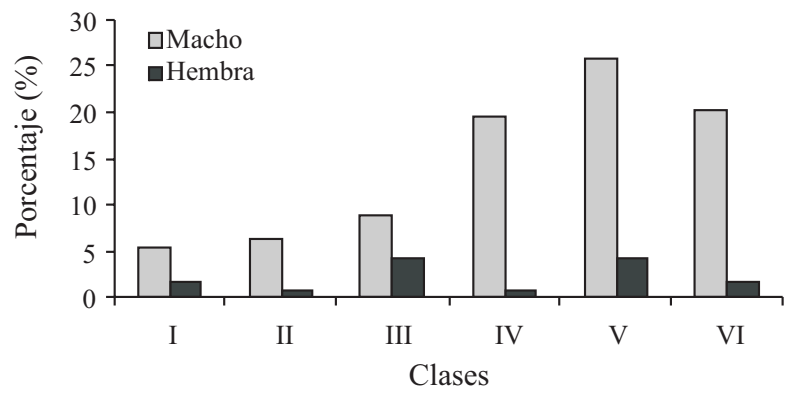

Fig. 2. Porcentaje de machos y hembras de Caiman crocodilus (Linnaeus, 1758) capturados en Caño Negro, Costa Rica ( $\mathrm{n}=113$ ).

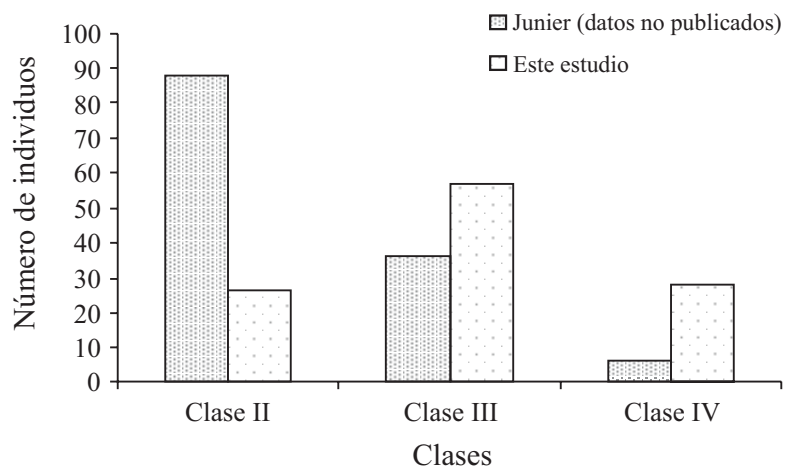

Fig. 3. Comparación del efecto de la caza ilegal de caimanes, Caiman crocodilus (Linnaeus, 1758), en este estudio (2004-2005) y los obtenidos anteriormente (1993-1994) (E. F. Junier, com. pers.) en la zona de Caño Negro, Costa Rica. 


\section{DISCUSIÓN}

Las diferencias en la estructura poblacional pudieron estar asociadas a factores climáticos como la temperatura del aire y del agua, velocidad del viento y período de luna. A pesar que no fueron medidas durante el estudio, se ha observado un efecto de las variables ambientales sobre el número de individuos observados de Crocodylus acutus (Cuvier, 1807) (Escobedo, 2003), Caiman yacare (Daudin, 1802), Melanosuchus niger (Spix, 1825) y Paleosuchus palpebrosus (Cuvier, 1807) (PAcheco, 1990; Coutinho \& CAmpos, 1996).

Las diferencias en el número de individuos por clase pudieron estar afectadas por los patrones de distribución espacial de la especie. Ouboter \& Nanhoe (1988) observaron que los individuos juveniles y sub-adultos tienden a ubicarse en zonas con una densa vegetación acuática como medida de protección; sin embargo, la presencia de vegetación entre el observador y el animal, produce un sesgo a la hora de determinar el número de individuos (COUTINHO \& CAMPOS, 1996).

En Caño Negro, los caimanes seleccionan sitios con troncos semi-sumergidos, ya que proveen protección y concentración de alimento (Allsteadt \& Vaughan, 1992), esto afecta el número de individuos capturados, ya que la presencia de troncos semi-sumergidos no es homogénea en tiempo y espacio, lo cual depende de la tasa de caída de árboles alrededor de los cuerpos de agua.

El porcentaje de individuos menores de $60 \mathrm{~cm}$ (Clase I) estuvo representado en su mayoría por neonatos, debido a que durante los primeros años de vida se mantienen cerca del área de anidación (SHAhrul \& Stuebing, 1996). Un alto porcentaje de individuos en los primeras etapas ontogenéticas ha sido observado en especies como Crocodylus acutus, C. moreletii Duméril \& Bibron, 1851, C. porosus Schneider, 1801 y C. yacare (Rebelo et al., 1997; Platt \& Thorbuarnarson, 2000; EsCoBEDO \& MeJ́́A, 2003; EsCOBEDO, 2005; EsPINAL, 2005). El bajo número de individuos de la clase II a la IV $(90$ y $150 \mathrm{~cm})$, puede deberse a que los individuos llegan a la madurez sexual en poco tiempo, debido al crecimiento exponencial durante esa etapa (Magnusson \& SanaiotiI, 1995). Por otro lado, el desplazamiento de juveniles y sub-adultos puede estar asociado a la estructura jerárquica dentro de la población para la época reproductiva.

Se observó un mayor número de machos en ambas épocas. Las diferencias en la proporción de sexos pueden estar asociadas a tres factores principales: primero, podría existir una diferencia en la selección de hábitat por parte de machos y hembras (THORBJARNARSON, 1997). E. F. Junier (com. pers.) observó en Caño Negro, que las hembras tienden a ubicarse en las lagunas mientras que los machos son más frecuentes sobre el cauce del río; sin embargo, las diferencias son cercanas a una proporción 1:1 macho/ hembra. Rebelo et al. (1997) observaron que la abundancia de machos y hembras de Caiman yacare es mayor en cuerpos de agua permanentes durante la época seca y disminuye durante la época lluviosa, siendo la proporción cercana 1:1 macho/hembra. Durante los 22 muestreos nocturnos en Caño Negro se abarcó el cauce principal del río Frío y las lagunas, para disminuir un posible dimorfismo en la selección de hábitat.
Segundo, probablemente la proporción de sexos esté influenciada directamente por la temperatura de incubación, siendo un reflejo a partir de la eclosión, ya que el caimán es una especie sexo dependiente de la temperatura de incubación (PIÑa et al., 2003). En Caño Negro, se observó un aumento en la temperatura de incubación en nidos de caimanes, siendo la temperatura sesgada hacia los machos (EscoBEDo, 2006).

Tercero, la cacería es uno de los posibles factores que podría estar afectando la proporción de sexos. Crawshaw (1990) mencionó que la disminución en el número de hembras está asociado a la cacería, debido a que durante la época de anidación las hembras tienden a ubicarse en las orillas de los cuerpos de agua para proteger los nidos, siendo visibles para los cazadores, a diferencia de los machos que se mantienen más tiempo en el agua. Sin embargo, ReBELo et al. (1997) observaron que la caza es selectiva sobre caimanes mayores a $1.2 \mathrm{~m}$ de longitud total y que el $60 \%$ de los individuos cazados son machos. La población de caimanes de Caño Negro ha sido sometida a una explotación desde hace varias décadas, principalmente para la comercialización de la piel a Nicaragua. En Caño Negro es difícil conocer la taza y proporción de machos y hembras de individuos cazados, dado que los cazadores después de extraer la piel de animal, se dan a la tarea de enterrar los cadáveres en fosas. Actualmente, uno de los factores que promueven la matanza de caimanes es la perspectiva negativa por parte de algunos de los pobladores de las comunidades dentro del refugio Caño Negro, por que el caimán se alimenta principalmente de peces como Sarotherodon aureus, Roeboides guatemalensis, Brycon guatemalensis, Cichlasoma dovii y $C$. citrinellum (Allsteadt \& Vaughan, 1994), que son fuente de ingresos y alimento para los pobladores de Caño Negro.

Asumiendo que el número de individuos capturados es representativo del tamaño y proporción de sexos de la población, se puede observar un número significativo de individuos dentro de la población reproductiva; sin embargo, la proporción de sexos de la población reproductiva es de 5.8:1 a 22:1 machos/ hembras. Esto puede traer consecuencias en la viabilidad poblacional a futuro, afectando el equilibro del humedal a mediano y largo plazo.

Agradecimientos. A Natalia Corrales Gómez, Fegmei Wu Chen y José González Maya por su apoyo en los recorridos de campo. A Maurizio Protti, Michael McCoy y José Retana por sus comentarios y sugerencias durante la realización de este estudio. A los funcionarios del Refugio Nacional de Vida Silvestre Caño Negro por su apoyo en la realización de este estudio.

\section{REFERENCIAS BIBLIOGRÁFICAS}

Allsteadt, J. \& Vaughan, C. 1992. Dry season habitat selection of Caiman crocodilus (Crocodylia: Alligatoridae) in Caño Negro, Costa Rica. Brenesia 38:65-69.

1994. Food habits of Caiman crocodilus in Caño Negro, Costa Rica. Vida Silvestre Neotropical 3:24-29.

Bolton, M. 1989. The management of crocodiles in captivity. Rome \& London, Food and Agriculture Organization. 62p.

Brazaitis, P. J. 1968. The determination of sex in living crocodilians. Herpetological Journal 4:54-58.

Cabrera, J.; Protti, M.; Urriola, M. \& Cubero, R. 2003. Distribución y abundancia de Caiman crocodilus en el Refugio Nacional de 
Vida Silvestre Caño Negro, Costa Rica. Revista de Biología Tropical 51:571-578.

Campos, Z.; Mourao, M.; Coutinho, M. \& Abercrombie, C. 1994. Night-light, size structures, and sex ratios in wild populations of yacare (Caiman crocodilus yacare) in the Brazilian Pantanal. Vida Silvestre Neotropical 4:46-50.

Castillo, R. \& March, J. 1993. Cambios en los hábitats ecológicos del Refugio Nacional de Vida Silvestre Caño Negro 19611992. Ciencias Sociales 62:51-67.

Continho, M. \& Campos, Z. 1996. Effect of habitat and seasonality on the densities of caiman in southern Brazil. Journal of Tropical Ecology 12:741-747.

Crawshaw, P. G. 1990. Effects of hunting on the reproduction of the Paraguayan Caiman (Caiman yacare) in the Pantanal of Mato Grosso, brazil. In: Robinson, J. G. \& Kent, H. eds. Redford Neotropical wildlife use and conservation. Chicago, The University of Chicago. p.145-153.

Escobedo, A. H. 2003. Períodos de actividad y efecto de las variables ambientales en cocodrilos (Crocodylus acutus Cuvier 1807): evaluando los métodos de determinación de la fracción visible. Ecología aplicada 2:136-140.

2005. El Lagarto Amarillo (Crocodylus acutus, Crocodylia: Crocodylidae): su estado poblacional en dos ríos del Pacífico Central de Costa Rica. Brenesia 63-64:113-120.

2006. Temperature variation in nests of Caiman crocodilus (Crocodylia: Alligatoridae). Acta Herpetologica 1(2):131-134.

Escobedo, A. H. \& Mejía, F. 2003. El "Cocodrilo de Tumbes" (Crocodylus acutus Cuvier 1807): estudio preliminar de su estado actual en el norte de Perú. Ecología aplicada 2:133-135.

Espinal, M. 2005. American crocodiles in Cajon Dam. Crocodile Specialist Group Newsletter 24:5-6.

Magnusson, W. E. \& SanaiotiI, T. M. 1995. Growth of Caiman crocodilus crocodilus in Central Amazonia, Brazil. Copeia 1995:498-501.

Mourao, G.; Campos, Z.; Coutinho, M. \& Abercrombie, C. 1996. Size structure of illegally harvested and surviving caiman Caiman crocodilus yacare in Pantanal, Brazil. Biological Conservation 75:261-265.
Ouboter, P. E. \& Nanhoe, L. M. R. 1988. Habitat selection and migration of Caiman crocodilus crocodilus in a Swamp and Swamp-Forest habitat in Northern Suriname. Journal of Herpetology 22:283-294.

PACHECO, L. 1990. Wariness of caiman populations and its effect on abundance estimates. Journal of Herpetology 30:123-126.

Piña, C. I.; Larriera, A. \& Cabrera, M. R. 2003. Effect of temperature on incubation period, sex ratio, hatching success and survivorship in Caiman latirostris (Crocodylia, Alligatoridae). Journal of Herpetology 37:199-202.

Platt, S. G. \& Thorbjarnarson, J. B. 2000. Population status and conservation of Morelet's crocodile, Crocodylus moreletii, in northern Belize. Biological Conservation 96:21-29.

Rebelo, G. H.; Nogueira, G. A.; Yamashita, C. \& Garcia de Arruba, A. 1997. Growth, sex ratio, population structure, and hunting mortality of Caiman yacare in the Pantanal, Brazil. Vida Silvestre Neotropical 6:29-36.

Ross, J. P. 1998. Crocodiles. Status survey and conservation action plan. $2^{\text {nd }}$ Edition. UICN/SSC. Gland, Switzerland and Cambridge, UK. Viii + 96p.

Sánchez, J.; Bolaños, J. \& Piedra, L. 1996. Población de Crocodylus acutus (Crocodylia: Crocodylidae) en dos ríos de Costa Rica. Revista de Biología Tropical 44:835-840.

Shahrul, A. M. S. \& Stuebing, R. B. 1996. Diet, growth and movements of juvenile crocodiles Crocodylus porosus Schneider in the Klias River, Sabah, Malaysia. Journal of Tropical Ecology 12:651-662.

ThorbJaRnarson, J. B. 1997. Are crocodilian sex ratios female biased? The data are equivocal. Copeia 1997:451-455.

Thorbuarnarson, J. B.; Mazzotti, F.; Sanderson, E.; Buitrago, F.; Lazcano, M.; Minkowski, K.; Muñiz, M.; Ponce, P.; Sigler, L.; Soberon, R.; Trelancia, A. M. \& Velasco, A. 2006. Regional habitat conservation priorities for the American crocodile. Biological Conservation 128:25-36.

Velasco, A. \& Ayarzaguena, J. 1995. Situación actual de las poblaciones de baba (Caiman crocodilus) sometidas a aprovechamiento comercial en los Llanos Venezolanos. Publicaciones de la Asociación Amigos Doñana 5:1-71.

Recebido em julho de 2006. Aceito em outubro de 2008. ISSN 0073-4721

Artigo disponível em: www.scielo.br/isz 